\title{
INSTABILITY AND STABILITY \\ OF NUMERICAL APPROXIMATIONS \\ TO DISCRETE VELOCITY MODELS \\ OF THE BOLTZMANN EQUATION
}

\author{
BY \\ ROBERT PESZEK \\ Department of Mathematical Sciences, Michigan Technological University, Houghton, MI
}

\begin{abstract}
We study a standard, explicit finite difference approximation of the 2-D Broadwell model and construct a numerical solution with the sum-norm growing in time faster than any polynomial. Our construction is based on a structure of a self-similar fractal!

We also obtain global existence, long-time behavior and numerical stability results of a large class of multidimensional discrete velocity models of the Boltzmann equation. We assume certain restrictions on the size of the support and the sup-norm of the initial data. Our results are obtained by examining the time evolution of sets on which the solutions are supported.
\end{abstract}

1. Introduction. In this paper we will study the $L^{\infty}$ stability of numerical solutions to the discrete velocity models of the Boltzmann equation. We will also present a global existence result for solutions of such models. The techniques presented in this paper are applicable to a quite large class of discrete velocity models, but to simplify the exposition we will focus our attention on the 2-D Broadwell model. This model is given by the following hyperbolic semilinear system of partial differential equations:

$$
\begin{aligned}
\frac{\partial}{\partial t} r(x, y, t)+\frac{\partial}{\partial x} r(x, y, t) & =u(x, y, t) d(x, y, t)-r(x, y, t) l(x, y, t), \\
\frac{\partial}{\partial t} l(x, y, t)-\frac{\partial}{\partial x} l(x, y, t) & =u(x, y, t) d(x, y, t)-r(x, y, t) l(x, y, t), \\
\frac{\partial}{\partial t} u(x, y, t)+\frac{\partial}{\partial y} u(x, y, t) & =r(x, y, t) l(x, y, t)-u(x, y, t) d(x, y, t), \\
\frac{\partial}{\partial t} d(x, y, t)-\frac{\partial}{\partial y} l(x, y, t) & =r(x, y, t) l(x, y, t)-u(x, y, t) d(x, y, t),
\end{aligned}
$$

with standard initial conditions imposed at time $t=0$

$$
(r, l, u, d)(x, y, 0)=\left(r_{0}, l_{0}, u_{0}, d_{0}\right)(x, y), \quad r_{0}, l_{0}, u_{0}, d_{0} \geq 0 .
$$

Received September 15, 1994.

1991 Mathematics Subject Classification. Primary 39A11, 35Q20, 35L45, 65M10.

(C)1996 Brown University 
Local existence results imply that the solution of (1.1) and (1.2) is defined on a strip $\{(x, y, t):-\infty<x<\infty,-\infty<y<\infty, 0 \leq t<T\}$ where $T$ depends on the size of the initial data. A difficult question, which we will examine, is if one can let $T=\infty$.

System (1.1) and other similar systems were studied by many authors. We refer the reader to the review paper of Platkowski and Illner [1] and the references contained therein for a survey of the theory of such systems. Equations (1.1) model the motion of an idealized gas of particles that can travel only with prescribed velocities. Equations (1.1) are simply the balance identities for such a gas where $r, l, u$, and $d$ represent the number (or the density) of particles that travel with velocities $(1,0),-(1,0),(0,1)$, and $-(0,1)$, and $\pm(u d-r l)$ are the collision terms.

In this paper we study the following explicit finite difference scheme:

$$
\begin{aligned}
R_{n, m}^{k} & =R_{n-1, m}^{k-1}+h Q_{n-1, m}^{k-1}, \\
L_{n, m}^{k} & =L_{n+1, m}^{k-1}+h Q_{n+1, m}^{k-1} \\
U_{n, m}^{k} & =U_{n, m-1}^{k-1}-h Q_{n, m-1}^{k-1}, \\
D_{n, m}^{k} & =D_{n, m+1}^{k-1}-h Q_{n, m+1}^{k-1},
\end{aligned}
$$

where $h=\Delta t=\Delta x=\Delta y$ is the mesh size, $(R, L, U, D)_{n, m}^{k}$ denotes the approximation of $(r, l, u, d)(n h, m h, k h)$, and $Q$ is the numerical collision term

$$
Q_{n, m}^{k}=U_{n, m}^{k} D_{n, m}^{k}-R_{n, m}^{k} L_{n, m}^{k}
$$

We define a sequence $\left(r^{h}, l^{h}, u^{h}, d^{h}\right)$ of piecewise constant functions by requiring that

$$
\begin{aligned}
& \left(r^{h}, l^{h}, u^{h}, d^{h}\right)(x, y, t)=\left(R_{n, m}^{k}, L_{n, m}^{k}, U_{n, m}^{k}, D_{n, m}^{k}\right) \quad \text { for } \\
& \quad n-\frac{1}{2}<x \leq n+\frac{1}{2}, \quad m-\frac{1}{2}<y \leq m+\frac{1}{2}, \quad k-\frac{1}{2}<t \leq k+\frac{1}{2},
\end{aligned}
$$

and assume that the numerical initial conditions

$$
\left(r^{h}, l^{h}, u^{h}, d^{h}\right)(x, y, 0)=\left(r_{0}^{h}, l_{0}^{h}, u_{0}^{h}, d_{0}^{h}\right)(x, y)
$$

are nonnegative, bounded uniformly in $L^{\infty}$, and satisfy

$$
\left(r_{0}^{h}, l_{0}^{h}, u_{0}^{h}, d_{0}^{h}\right) \stackrel{h \rightarrow 0}{\rightarrow}\left(r_{0}, l_{0}, u_{0}, d_{0}\right) \text { in } L_{\mathrm{loc}}^{p} .
$$

We note that solutions of (1.1) are nonnegative if the initial data is nonnegative, but it is not so for solutions of $(1.3) .^{1}$

As was pointed out by Peszek [5], if for $0 \leq t \leq T$ the sequence $\left(r^{h}, l^{h}, u^{h}, d^{h}\right)$ is bounded uniformly in $L^{\infty}$, then the unique solution $(r, l, u, d)$ of $(1.1)$ and (1.2) exists on the time interval $t \in[0, T]$, and

$$
\left(r^{h}, l^{h}, u^{h}, d^{h}\right) \rightarrow(r, l, u, d) \text { in } L_{\mathrm{loc}}^{p}, \quad 1 \leq p<\infty
$$

\footnotetext{
${ }^{1}$ However, if the sequence of solutions to (1.3) is bounded uniformly from above as $h \rightarrow 0$ and if the numerical initial data is nonnegative, then for sufficiently small $h$ these solutions remain nonnegative at later times $t$.
} 
One would like, thus, to ask if the numerical scheme (1.3) is $L^{\infty}$ stable for $t \in[0, \infty)$, i.e., if

$$
\left\|r_{0}^{h}\right\|_{\infty} \leq M(0), \quad\left\|l_{0}^{h}\right\|_{\infty} \leq M(0), \quad\left\|u_{0}^{h}\right\|_{\infty} \leq M(0), \quad\left\|d_{0}^{h}\right\|_{\infty} \leq M(0)
$$

implies

$$
\max \left(\left\|r^{h}(\cdot, \cdot, t)\right\|_{\infty},\left\|l^{h}(\cdot, \cdot, t)\right\|_{\infty},\left\|u^{h}(\cdot, \cdot, t)\right\|_{\infty},\left\|d^{h}(\cdot, \cdot, t)\right\|_{\infty}\right) \leq M(t)
$$

for some function $M:[0, \infty] \rightarrow(0, \infty)$ independent of $h$. Local $L^{\infty}$ stability is easily shown by taking

$$
M(t)=\frac{M(0)}{1-M(0) t} .
$$

The observation that both numerical and exact solutions of (1.1) are bounded by a function $M$ that satisfies $\frac{d}{d t} M(t)=M(t)^{2}$ gives local $L^{\infty}$ stability with (1.11). It is substantially more complicated to obtain a function $M$ defined on $[0, \infty)$.

One can easily construct both exact and numerical solutions for which the $L^{\infty}$ norm grows linearly or quadratically in time (see [3]). Thus $M(t)$ has to grow faster than $O\left(t^{2}\right)$. The main result of this paper shows that the numerical scheme (1.3) is not polynomially $L^{\infty}$ stable, that is, that the above function $M$ has to grow faster than any polynomial. This result is based on fractal-like constructions of motions that satisfy (1.3). Constructed numerical solutions satisfy

$$
\begin{gathered}
\left(r_{0}^{h}, l_{0}^{h}, u_{0}^{h}, d_{0}^{h}\right) \stackrel{h \rightarrow 0}{\rightarrow} 0, \quad \text { in } L_{\text {loc }}^{p}, 1 \leq p<\infty \\
\left\|r^{h}(\cdot, \cdot, 0)\right\|_{\infty}=\left\|l^{h}(\cdot, \cdot, 0)\right\|_{\infty}=\left\|u^{h}(\cdot, \cdot, 0)\right\|_{\infty}=\left\|d^{h}(\cdot, \cdot, 0)\right\|_{\infty}=\varepsilon
\end{gathered}
$$

where $\varepsilon>0$ is a given constant, and

$$
\left\|r^{h}\left(\cdot, \cdot, 2^{s} h\right)\right\|_{\infty}>\left\|r^{h}\left(\cdot, \cdot, 2^{s-1} h\right)\right\|_{\infty}+h\left\|r^{h}\left(\cdot, \cdot, 2^{s-1} h\right)\right\|_{\infty}^{2}
$$

and, thus, produce a growth that is faster than any polynomial.

Our construction is based on the ordered graph shown in Fig. 1. This graph has the fractal structure. The initial data is supported on a set based on a set which approximates a fractal shown in Fig. 2. This fractal has 2-Lebesgue measure 0, and this is the basis of proving (1.12). The idea of using fractals to study PDEs is not new (cf. Mandelbrot $[6])$, but this is probably the first time that a self-similar structure of a fractal is used to study the stability of finite difference schemes.

Our construction suggests that one may need to impose some additional restrictions on initial data to guarantee the stability. It turns out that global $L^{\infty}$ stability is closely related to obtaining an a priori $L^{\infty}$ estimate for the exact solution of (1.1). Such estimates automatically give global existence of solutions to the Broadwell model. Again we refer the reader to the review paper of Platkowski and Illner for the survey of existence results. In particular, a priori $L^{\infty}$ estimates for the 3-D Broadwell model were obtained by Kawashima [4] and then generalized to a class of other models by Illner [2]. Their 
methods require the initial data to be small in suitable norms and can be reformulated as a stability result for the numerical scheme (1.3).

In the last section of this paper we will present a global existence and a stability result for the Broadwell model (1.1). We will assume that the support of the initial data is restricted to one of the strips

$$
\Omega_{1}=\{(x, y):|x-y| \leq a\},
$$

or

$$
\Omega_{2}=\{(x, y):|x+y| \leq a\},
$$

and that the size $a$ of the strip is small compared to $M_{0}:=\max \left(\left\|r_{0}\right\|_{\infty},\left\|l_{0}\right\|_{\infty},\left\|u_{0}\right\|_{\infty}\right.$, $\left.\left\|d_{0}\right\|_{\infty}\right)$

$$
a M_{0}<2 .
$$

Let us restrict our attention to the case (1.15). Global existence follows from the observation that the support of $(r+d)(\cdot, \cdot, t)$ travels southeast and is included in the set $\{(x, y):|x-y-t| \leq a\}$ while the support of $(l+u)(\cdot, \cdot, t)$ travels northwest and is included in the set $\{(x, y):|x-y+t| \leq a\}$. For times $t \geq a / 2$ the supports are disjoint and the functions $r, l, u$, and $d$ become constant along corresponding characteristics. Now one can employ the estimate (1.11) to guarantee that the time of separation $t_{\text {sep }}=a / 2$ occurs before the blow-up time $t_{\text {crit }}=1 / N(0)$ and, thus, to guarantee global existence. This method can be generalized to a large class of multidimensional discrete velocity models of the Boltzmann equation yielding global existence and long-time behavior results. The admissible class of models includes the full 3-D Broadwell system, as well as the coplanar model with $2 r$ velocities (see [1] for definitions). As we will show in the end of the last section, the diagonality of the choices for the strips $\Omega_{1}$ and $\Omega_{2}$ in the above discussion is not essential.

We note that, in the same way, one obtains the corresponding $L^{\infty}$ stability bound for the numerical solutions. These results are apparently new; at least we have not seen them in the literature.

2. An instability result and fractals. In this section we shall study the numerical scheme

$$
\begin{aligned}
& R_{n, m}^{k}=R_{n-1, m}^{k-1}+h Q_{n-1 . m}^{k-1}, \\
& L_{n . m}^{k}=L_{n+1 . m}^{k-1}+h Q_{n+1 . m}^{k-1}, \\
& U_{n, m}^{k}=U_{n, m-1}^{k-1}-h Q_{n, m-1}^{k-1}, \\
& D_{n, m}^{k}=D_{n, m+1}^{k-1}-h Q_{n . m+1}^{k-1},
\end{aligned}
$$

where $h=\Delta t=\Delta x=\Delta y$ is the mesh size, $(R, L, U, D)_{n, m}^{k}$ denotes the approximation of $(r, l, u, d)(n h, m h, k h)$, and $Q$ is the numerical collision term,

$$
Q_{n, m}^{k}=U_{n, m}^{k} D_{n, m}^{k}-R_{n, m}^{k} L_{n, m}^{k} .
$$

The above finite difference scheme is defined on the set of grid points $G_{h}$,

$$
G_{h}=\left\{\left(x_{n}, y_{m}, t_{k}\right): \begin{array}{l}
x_{n}=n h, \quad y_{m}=m h, \quad t_{k}=k h, \\
\text { where } m, n, \text { and } k \text { are integers, and } 0 \leq k \leq T / h
\end{array}\right\} .
$$


As in the previous section, we introduce the following sequence of piecewise constant functions:

$$
\begin{aligned}
& \left(r^{h}, l^{h}, u^{h}, d^{h}\right)(x, y, t)=\left(R_{n, m}^{k}, L_{n . m}^{k}, U_{n, m}^{k}, D_{n, m}^{k}\right) \quad \text { for } \\
& \quad n-\frac{1}{2}<x \leq n+\frac{1}{2}, \quad m-\frac{1}{2}<y \leq m+\frac{1}{2}, \quad k-\frac{1}{2}<t \leq k+\frac{1}{2},
\end{aligned}
$$

and assume that the given numerical initial conditions

$$
\left(r^{h}, l^{h}, u^{h}, d^{h}\right)(x, y, 0)=\left(r_{0}^{h}, l_{0}^{h}, u_{0}^{h}, d_{0}^{h}\right)(x, y)
$$

are bounded uniformly in $L^{\infty}$. In this section we study the stability of the numerical scheme (2.1), and we will fix $h$ as a small parameter rather than considering $h \rightarrow 0$.

The numerical scheme (2.1) inherits the physical interpretation from the Broadwell model: we can think of trains of particles that travel with speed one along vertical and horizontal lines connecting the lattice $G_{h}$. Since they are initially positioned at the lattice locations collisions will happen at times $t_{k}=k h$, when the particle trains are again at the grid locations. The numerical scheme employs the same collision rule as the Broadwell equations; we simply assume that collisions occur only between particles moving right and left and between particles moving up and down and that each collision results in a change of velocity directions, which affects some number of particles. Right after the collision these particles will split into two groups moving in two directions (perpendicular to the direction before the collision). The number of particles affected in this change is assumed to be proportional to the product of colliding quantities multiplied by a factor $h$. We make our measurements of the motion only at grid points and only at specified times $t=k h$. Thus we will see the effect of the collision with the time lapse $h$, and this is exactly the behavior modeled by the scheme (2.1).

Now we are in the position to describe our construction. We fix $h$ and want to construct a numerical solution $\left(r^{h}, l^{h}, u^{h}, d^{h}\right)$ which exhibits a very fast time growth of $r^{h}$. We assume that the initial data $\left(r_{0}^{h}, l_{0}^{h}, u_{0}^{h}, d_{0}^{h}\right)$ satisfies

$$
\left\|r^{h}(\cdot, \cdot, 0)\right\|_{\infty}=\left\|l^{h}(\cdot, \cdot, 0)\right\|_{\infty}=\left\|u^{h}(\cdot, \cdot, 0)\right\|_{\infty}=\left\|d^{h}(\cdot, \cdot, 0)\right\|_{\infty}=\varepsilon .
$$

The motion is described by the fractal-like ordered graph shown in Fig. 1 (see p. 782).

We let $r_{0}^{h}=l_{0}^{h}=u_{0}^{h}=d_{0}^{h}=0$ at all grid points $(n h, 0), n>0$ and also at points $(n, m)$ with $m>n$ or $m<-n$. We assign $r_{0}^{h}(0,0)=\varepsilon, u_{0}^{h}(h,-h)=\varepsilon$, and $d_{0}^{h}(h, h)=\varepsilon$. We assume that these are the only nonzero initial conditions in the square $K_{1}$, with vertices $(0,0),(h, h),(h,-h)$, and $(2 h, 0)$, except for $r_{0}^{h}(h, h)$ and $r_{0}^{h}(h,-h)$ which we will prescribe in the next step of our construction. Solving $(2.1)$ for $r^{h}(2 h, 0,2 h)$ yields

$$
r^{h}(2 h, 0,2 h)=r^{h}(h, 0, h)+h \varepsilon^{2}=r^{h}(h, 0, h)+h\left(r^{h}(h, 0, h)\right)^{2} .
$$

We observe that our initial data, as well as the solution, can be described by drawing a graph as in Fig. 1. If at a certain point one of the functions $r^{h}, l^{h}, u^{h}$, or $d^{h}$ is nonzero we will draw an arrow at this point. We will draw an arrow pointing to the right if $r^{h}$ is nonzero, an arrow pointing to the left if $l^{h}$ is nonzero and so on. The numbers on the graph refer to the times in which $r^{h}, l^{h}, u^{h}$, or $d^{h}$ is nonzero as described by the arrow. 


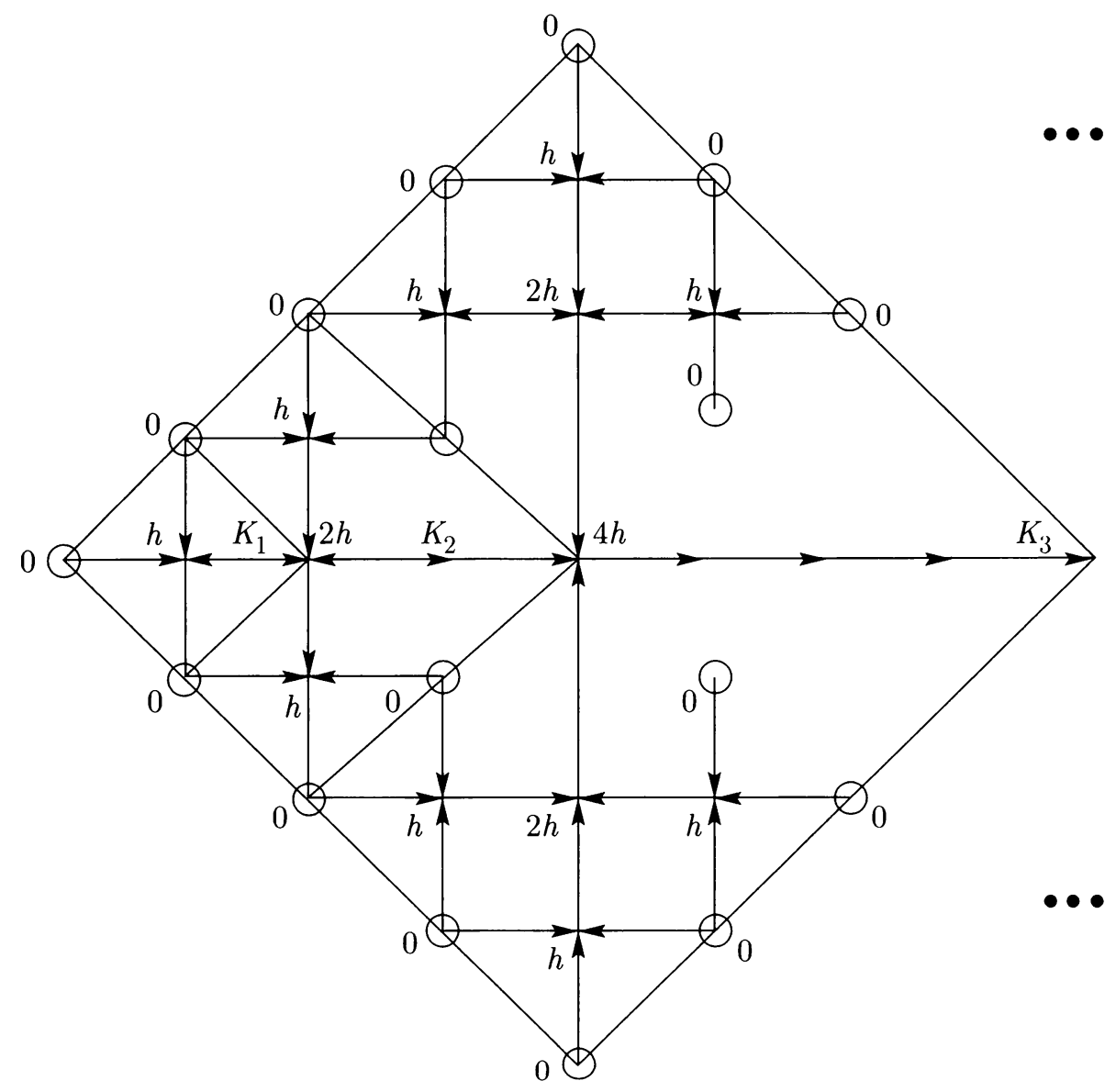

FIGURE 1. Numbers $0, h, 2 h$, and $4 h$ mark times in which the arrows are drawn. The symbol $\bigcirc$ marks all the points in the square $K_{3}$ at which the initial data is nonzero.

The basic trick is to rotate the pattern from the square $K_{1}$ around the point $(2 h, 0)$ by $90^{\circ}$ clockwise and counterclockwise. The term "rotating" refers of course to the arrows. After rotating clockwise $r^{h}$ starts to play the role of $u^{h}, l^{h}$ plays the role of $d^{h}$, and so on. The counterclockwise rotation requires that we substitute $r^{h}$ for $d^{h}$ and $l^{h}$ for $u^{h}$ and so on. Thus, the construction yields that

$$
d^{h}(2 h, 0,2 h)=u^{h}(2 h, 0,2 h)=r^{h}(2 h, 0,2 h)
$$

and, thus, that

$$
\begin{aligned}
r^{h}(3 h, 0,3 h) & =r^{h}(2 h, 0,2 h)+h u^{h}(2 h, 0,2 h) d^{h}(2 h, 0,2 h) \\
& =r^{h}(2 h, 0,2 h)+h\left(r^{h}(2 h, 0,2 h)\right)^{2} .
\end{aligned}
$$

Now we define the initial data on the remaining part of the square $K_{2}$, which has the vertices $(0,0),(2 h, 2 h),(2 h,-2 h)$, and $(4 h, 0)$. We simply put all initial data to be 0 , 
except for $r_{0}^{h}$ and $u_{0}^{h}$ on the northeast side of $K_{2}$ and for $r_{0}^{h}$ and $d_{0}^{h}$ on the southeast side of $K_{2}$. These values will be prescribed in the next step of our construction.

We repeat the above procedure by rotating the pattern $K_{2}$ around the point $(4 h, 0)$ by $90^{\circ}$ clockwise and counterclockwise, in the way that yields that

$$
d^{h}(4 h, 0,4 h)=u^{h}(4 h, 0,4 h)=r^{h}(4 h, 0,4 h)
$$

and that

$$
r^{h}(5 h, 0,5 h)=r^{h}(4 h, 0,4 h)+h r^{h}(4 h, 0,4 h)^{2} .
$$

This defines our solution on the square $K_{3}$ with vertices $(0,0),(4 h, 4 h),(4 h,-4 h)$, and $(8 h, 0)$. We observe that throughout the construction $r^{h}$ increases rapidly at points $r^{h}\left(2^{s} h, 0,2^{s} h\right)$ and is nondecreasing elsewhere on the line $y=0$.

Now let us assume that the initial data is defined on the square $K_{s}, s \geq 1$, with vertices $(0,0),\left(2^{s-1} h, 2^{s-1} h\right),\left(2^{s-1} h,-2^{s-1} h\right)$, and $\left(2^{s} h, 0\right)$, except for $r_{0}^{h}$ and $u_{0}^{h}$ on the northeast side of $K_{s}$ and for $r_{0}^{h}$ and $d_{0}^{h}$ on the southeast side of $K_{s}$. As above we rotate $K_{s}$ around the point $\left(2^{s} h, 0\right)$ by $90^{\circ}$ clockwise and counterclockwise, and the self-similarity of the graph yields that

$$
d^{h}\left(2^{s} h, 0,2^{s} h\right)=u^{h}\left(2^{s} h, 0,2^{s} h\right)=r^{h}\left(2^{s} h, 0,2^{s} h\right)
$$

and, thus, that

$$
r^{h}\left(\left(2^{s}+1\right) h, 0,\left(2^{s}+1\right) h\right)=r^{h}\left(2^{s} h, 0,2^{s} h\right)+h\left(r^{h}\left(2^{s} h, 0,2^{s} h\right)\right)^{2} .
$$

This recursive construction yields a numerical solution with the property that $r^{h}(k h, 0, k h)$ is nondecreasing. Thus, we obtain the following

LEMMA 2.1.

$$
r^{h}\left(2^{s+1} h, 0,2^{s+1} h\right) \geq r^{h}\left(2^{s} h, 0,2^{s} h\right)+h r^{h}\left(2^{s} h, 0,2^{s} h\right)^{2} \quad \text { for } s=0,1,2, \ldots
$$

DEFINITION. We say that the numerical scheme is polynomially stable if there exists a polynomial $P, P(t)>0$ for $t \geq 0$, with the property that for all initial conditions $\left(r_{0}^{h}, l_{0}^{h}, u_{0}^{h}, d_{0}^{h}\right)$ satisfying

$$
\left\|r_{0}^{h}\right\|_{\infty} \leq P(0), \quad\left\|l_{0}^{h}\right\|_{\infty} \leq P(0), \quad\left\|u_{0}^{h}\right\|_{\infty} \leq P(0), \quad\left\|d_{0}^{h}\right\|_{\infty} \leq P(0)
$$

one obtains

$$
\max \left(\left\|r^{h}(\cdot, \cdot, t)\right\|_{\infty},\left\|l^{h}(\cdot, \cdot, t)\right\|_{\infty},\left\|u^{h}(\cdot, \cdot, t)\right\|_{\infty},\left\|d^{h}(\cdot, \cdot, t)\right\|_{\infty}\right) \leq P(t)
$$

ThEOREM 2.1. The scheme $(2.1)$ is not polynomially stable.

Proof. We apply the above construction with $\varepsilon=P(0)$. We observe that after at most $s_{0}=\left[(1 / \varepsilon)^{2}(1 / h)^{2}\right]$ steps $s, r^{h}\left(2^{s} h, 0,2^{s} h\right) \geq 1 /\left(h e^{k h}\right)$ for some $k>0$. We also note that

$$
\gamma_{k}(p) \stackrel{\text { def }}{=}(1 / h) \exp (k p)
$$




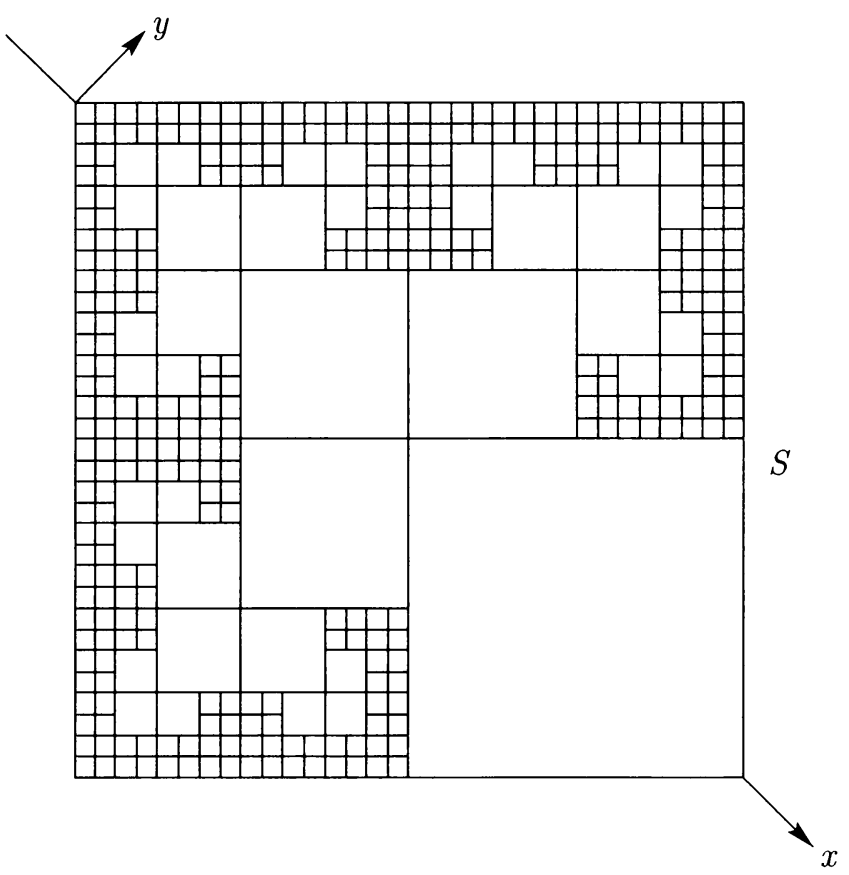

FIGURE 2. The initial data is supported on a set which approximates a self-similar fractal. The reader should rotate this picture by $45^{\circ}$ so that the coordinate system has its usual position. The fractal is obtained by the following procedure: Start with a square $S$ as shown on the picture and divide it into 4 equal squares. Remove the one closest to the right and mark the center of $S$. Divide the remaining squares into four equal squares each and remove squares closest to the marked point. Now mark centers of these 3 squares and repeat the procedure for each of the smallest squares. Repeat this procedure several times. If we stop when the remaining squares have diameter $2 h$, then they will tightly cover the part of the support of $\left(r_{0}^{h}, l_{0}^{h}, u_{0}^{h}, d_{0}^{h}\right)$ that is restricted to $S$. If we do not stop, we will obtain a self-similar fractal of Lebesque measure 0 .

satisfies

$$
\gamma_{k}\left(2^{s+1} h\right) \leq \gamma_{k}\left(2^{s} h\right)+h \gamma_{k}\left(2^{s} h\right)^{2} \quad \text { for } s=0,1,2, \ldots
$$

Thus, Lemma 2.1 implies that

$$
r^{h}\left(2^{s} h, 0,2^{s} h\right) \geq \gamma_{k}\left(2^{s-s_{0}} h\right),
$$

for $s \geq s_{0}$.

Now to conclude the proof we need only to note that $\gamma_{k}$ grows faster than any polynomial.

Our final observation is that, since the fractal associated with the support of $\left(r_{0}^{h}, l_{0}^{h}, u_{0}^{h}, d_{0}^{h}\right)$ has 2-Lebesgue measure 0 ,

$$
\left(r_{0}^{h}, l_{0}^{h}, u_{0}^{h}, d_{0}^{h}\right) \rightarrow 0 \quad \text { in } L_{\mathrm{loc}}^{p}, 1 \leq p<\infty .
$$


3. Remarks on numerical stability. It may seem that the construction described in the last section is sufficiently universal to obtain instability results similar to the one in Theorem 2.1 for other numerical schemes. Fortunately, this is not so. For example, let us consider the following implicit scheme:

$$
\begin{aligned}
R_{n, m}^{k} & =R_{n-1, m}^{k-1}+h Q_{n, m}^{k}, \\
L_{n, m}^{k} & =L_{n+1, m}^{k-1}+h Q_{n, m}^{k}, \\
U_{n, m}^{k} & =U_{n, m-1}^{k-1}-h Q_{n, m}^{k}, \\
D_{n, m}^{k} & =D_{n, m-1}^{k-1}-h Q_{n, m}^{k},
\end{aligned}
$$

where again $h=\Delta t=\Delta x=\Delta y$ is the mesh size, $(R, L, U, D)_{n, m}^{k}$ denotes the approximation of $(r, l, u, d)(n h, m h, k h)$, and $Q_{n, m}^{k}$ is the numerical collision term,

$$
Q_{n, m}^{k}=U_{n, m}^{k} D_{n, m}^{k}-R_{n, m}^{k} L_{n, m}^{k} .
$$

As in previous sections we let $\left(r^{h}, l^{h}, u^{h}, d^{h}\right)$ be the piecewise constant function that coincides with the finite difference solution $(R, L, U, D)$ on the grid points.

Scheme (3.1) turns out to be more stable; in particular, one obtains a priori bounds on the numerical solution of the order $O(1 / h)$. Thus, one cannot obtain an unbounded growth of the numerical solution for a fixed $h$ and we have to let $h \rightarrow 0$. If we repeat the construction from the above section we will obtain the following result.

Let $h$ be small. Then as long as

$$
h\left(\max _{p \in N, p h \leq T}\left(u^{h}(p h, 0, p h), r^{h}(p h, 0, p h)\right)\right)<1 / 2
$$

one obtains

$$
\begin{array}{r}
r^{h}\left(2^{s+1} h, 0,2^{s+1} h\right) \geq r^{h}\left(2^{s} h, 0,2^{s} h\right)+\frac{2}{9} h\left(r^{h}\left(2^{s} h, 0,2^{s} h\right)\right)^{2} \\
\text { for } s \text { such that } 2^{s+1} h \leq T .
\end{array}
$$

To obtain (3.4) we note that the implicit character of the scheme (3.1) introduces some changes in the argument from the last section. The basic change is that one has to replace the equation (2.9) by

$$
r^{h}\left(\left(2^{s}-1\right) h, 0,\left(2^{s}-1\right) h\right)=u^{h}\left(\left(2^{s}-1\right) h, 0,\left(2^{s}-1\right) h\right)=d^{h}\left(\left(2^{s}-1\right) h, 0,\left(2^{s}-1\right) h\right) .
$$

Now to obtain $r^{h}\left(2^{s} h, 0,2^{s} h\right)$ we have to deal with a more complicated implicit system of equations (3.1). This system reduces to ${ }^{2}$

$$
\begin{aligned}
r^{h}\left(2^{s} h, 0,2^{s} h\right)= & r^{h}\left(\left(2^{s}-1\right) h, 0,\left(2^{s}-1\right) h\right)+h\left(u^{h}\left(2^{s} h, 0,2^{s} h\right)\right)^{2} \\
& -h r^{h}\left(2^{s} h, 0,2^{s} h\right) l^{h}\left(2^{s} h, 0,2^{s} h\right), \\
l^{h}\left(2^{s} h, 0,2^{s} h\right)= & h\left(u^{h}\left(2^{s} h, 0,2^{s} h\right)\right)^{2}-h r^{h}\left(2^{s} h, 0,2^{s} h\right) l^{h}\left(2^{s} h, 0,2^{s} h\right), \\
d^{h}\left(2^{s} h, 0,2^{s} h\right)= & u^{h}\left(2^{s} h, 0,2^{s} h\right), \\
u^{h}\left(2^{s} h, 0,2^{s} h\right) \geq & r^{h}\left(\left(2^{s}-1\right) h, 0,\left(2^{s}-1\right) h\right)-h\left(u^{h}\left(2^{s} h, 0,2^{s} h\right)\right)^{2} .
\end{aligned}
$$

\footnotetext{
${ }^{2} \mathrm{~A}$ straightforward, inductive argument shows that $r(p h, 0, p h)>0$ and $u(p h, 0, p h)=d(p h, 0, p h)>0$ for all $p>0$.
} 
Plugging $(3.6)_{2}$ into $(3.6)_{1}$ and using (3.3) yields

$$
r^{h}\left(2^{s} h, 0,2^{s} h\right) \geq r^{h}\left(\left(2^{s}-1\right) h, 0,\left(2^{s}-1\right) h\right)+\frac{1}{2} h\left(u^{h}\left(2^{s} h, 0,2^{s} h\right)\right)^{2},
$$

while applying (3.3) to $(3.6)_{4}$ yields that $u^{h}\left(2^{s} h, 0,2^{s} h\right) \geq(2 / 3) r^{h}\left(\left(2^{s}-1\right) h, 0,\left(2^{s}-1\right) h\right)$. Now a brief look is enough to verify that

$$
r^{h}\left(2^{s} h, 0,2^{s} h\right) \geq r^{h}\left(\left(2^{s}-1\right) h, 0,\left(2^{s}-1\right) h\right)+\frac{2}{9} h\left(r^{h}\left(\left(2^{s}-1\right) h, 0,\left(2^{s}-1\right) h\right)\right)^{2} .
$$

To show (3.4) we need to prove that $r^{h}(p h, 0, p h)$ is a nondecreasing function of $p$. The calculation which shows that is similar to the one above, and therefore omitted.

Condition (3.4) shows that the $L^{\infty}$ norm of the numerical solution diverges as $h \rightarrow 0$. It turns out, however, that the time needed for this solution to attain a rapid growth is $O(1 / h)$ or larger and, thus, we cannot claim at this point that the scheme (3.1) is not polynomially stable.

4. Global existence results. In this section we will show a global existence result for solutions to a large class of discrete velocity models of the Boltzmann equation. The results presented here are quite elementary and simple in the context of the 2-D Broadwell model,

$$
\begin{gathered}
\frac{\partial}{\partial t} r+\frac{\partial}{\partial x} r=u d-r l \\
\frac{\partial}{\partial t} l-\frac{\partial}{\partial x} l=u d-r l \\
\frac{\partial}{\partial t} u+\frac{\partial}{\partial y} u=r l-u d, \\
\frac{\partial}{\partial t} d-\frac{\partial}{\partial y} d=r l-u d .
\end{gathered}
$$

To clarify our exposition we start from the model system (4.1). At the end of this section we will discuss how our method is applied to a more general situation.

We restrict our attention to the case in which the support of the initial data

$$
\left(r_{0}, l_{0}, u_{0}, d_{0}\right)(x, y)
$$

is included in one of the strips

$$
\Omega_{1}(a)=\{(x, y):|x-y| \leq a\} \quad \text { or } \quad \Omega_{2}(a)=\{(x, y):|x+y| \leq a\} .
$$

For the sake of generality we consider nonnegative solutions to the more general system

$$
\begin{aligned}
\frac{\partial}{\partial t} u_{1}(x, y, t)+\frac{\partial}{\partial x} u_{1}(x, y, t) & \leq u_{3}(x, y, t) u_{4}(x, y, t), \\
\frac{\partial}{\partial t} u_{2}(x, y, t)-\frac{\partial}{\partial x} u_{2}(x, y, t) & \leq u_{3}(x, y, t) u_{4}(x, y, t), \\
\frac{\partial}{\partial t} u_{3}(x, y, t)+\frac{\partial}{\partial y} u_{3}(x, y, t) & \leq u_{1}(x, y, t) u_{2}(x, y, t), \\
\frac{\partial}{\partial t} u_{4}(x, y, t)-\frac{\partial}{\partial y} u_{4}(x, y, t) & \leq u_{1}(x, y, t) u_{2}(x, y, t)
\end{aligned}
$$

and prove the following: 
Theorem 4.1. Assume that $u_{1}, u_{2}, u_{3}$, and $u_{4}$ are uniformly bounded, nonnegative functions defined on $\{(x, y, t): 0 \leq t \leq T\}$, and satisfying (4.4). Assume also that

$$
\operatorname{supp}\left[\left(u_{1}, u_{2}, u_{3}, u_{4}\right)(\cdot, \cdot, 0)\right] \subset \Omega_{1}(a)
$$

Then for $0 \leq t \leq T$

$$
\operatorname{supp}\left[\left(u_{1}, u_{4}\right)(\cdot, \cdot, 0)\right] \subset\{(x, y):|x-y-t| \leq a\}=\Omega_{1}(a)+(t, 0)=\Omega_{1}(a)+(0,-t)
$$

and

$$
\operatorname{supp}\left[\left(u_{2}, u_{3}\right)(\cdot, \cdot, t)\right] \subset\{(x, y):|x-y+t| \leq a\}=\Omega_{1}(a)+(-t, 0)=\Omega_{1}(a)+(0, t)
$$

In particular, all times $t \geq a / 2$, supports are disjoint and $u_{1}, u_{2}, u_{3}$, and $u_{4}$ satisfy

$$
\begin{aligned}
\frac{\partial}{\partial t} u_{1}(x, y, t)+\frac{\partial}{\partial x} u_{1}(x, y, t) & \leq 0 \\
\frac{\partial}{\partial t} u_{2}(x, y, t)-\frac{\partial}{\partial x} u_{2}(x, y, t) & \leq 0 \\
\frac{\partial}{\partial t} u_{3}(x, y, t)+\frac{\partial}{\partial y} u_{3}(x, y, t) & \leq 0 \\
\frac{\partial}{\partial t} u_{4}(x, y, t)-\frac{\partial}{\partial y} u_{4}(x, y, t) & \leq 0
\end{aligned}
$$

Similarly, if

$$
\operatorname{supp}\left[\left(u_{1}, u_{2}, u_{3}, u_{4}\right)(\cdot, \cdot, 0)\right] \subset \Omega_{2}(a)
$$

then

$$
\operatorname{supp}\left[\left(u_{1}, u_{3}\right)(\cdot, \cdot, t)\right] \subset\{(x, y):|x+y-t| \leq a\}=\Omega_{2}(a)+(t, 0)=\Omega_{2}(a)+(0, t)
$$

and

$$
\operatorname{supp}\left[\left(u_{2}, u_{4}\right)(\cdot, \cdot, t)\right] \subset\{(x, y):|x+y+t| \leq a\}=\Omega_{2}(a)-(t, 0)=\Omega_{2}(a)-(0, t)
$$

Before proving this theorem we want to make a few observations. Let

$$
M(t) \stackrel{\text { def }}{=} \max \left(\left\|u_{1}(\cdot, \cdot, t)\right\|_{\infty},\left\|u_{2}(\cdot, \cdot, t)\right\|_{\infty},\left\|u_{3}(\cdot, \cdot, t)\right\|_{\infty},\left\|u_{4}(\cdot, \cdot, t)\right\|_{\infty}\right) .
$$

Equations (4.4) yield that $\frac{d}{d t} M \leq M^{2}$ and, thus, that

$$
M(t) \leq \frac{M(0)}{1-M(0) t}
$$

This observation, together with Theorem 4.1, yields the following global existence result for the 2-D Broadwell model. 
Corollary 4.1. Assume that the initial data is nonnegative and satisfies

$$
\begin{gathered}
\left\|r_{0}\right\|_{\infty},\left\|l_{0}\right\|_{\infty},\left\|u_{0}\right\|_{\infty},\left\|d_{0}\right\|_{\infty} \leq M_{0}, \\
\operatorname{supp}\left(r_{0}, l_{0}, u_{0}, d_{0}\right) \subset \Omega_{1}(a) \quad\left(\text { or } \subset \Omega_{2}(a)\right),
\end{gathered}
$$

and

$$
M_{0} a<2 .
$$

Then the (unique) globally defined solution $(r, l, u, d)$ to (4.1) and (1.2) exists. Moreover, $(r, l, u, d)$ satisfy

$$
\begin{aligned}
\frac{\partial}{\partial t} r(x, y, t)+\frac{\partial}{\partial x} r(x, y, t) & =0 \\
\frac{\partial}{\partial t} l(x, y, t)-\frac{\partial}{\partial x} l(x, y, t) & =0 \\
\frac{\partial}{\partial t} u(x, y, t)+\frac{\partial}{\partial y} u(x, y, t) & =0 \\
\frac{\partial}{\partial t} d(x, y, t)-\frac{\partial}{\partial y} d(x, y, t) & =0
\end{aligned}
$$

for $t \geq a / 2$.

The following is also a trivial consequence of Theorem 4.1.

Corollary 4.2. Assume that $(r, l, u, d)$ is a uniformly bounded solution of $(4.1)$ defined on $\{(x, y, t): t \geq 0\}$ and that the initial data is nonnegative and has bounded support.

Then, for sufficiently large $a$, the support is included in the rectangle $K(a)=\Omega_{1}(a) \cap$ $\Omega_{2}(a)$

$$
\operatorname{supp}\left(r_{0}, l_{0}, u_{0}, d_{0}\right) \subset K(a)
$$

and

$$
\begin{aligned}
& \operatorname{supp}(r(\cdot, \cdot, t)) \subset K(a)+(t, 0), \\
& \operatorname{supp}(l(\cdot, \cdot, t)) \subset K(a)+(-t, 0), \\
& \operatorname{supp}(u(\cdot, \cdot, t)) \subset K(a)+(0, t), \\
& \operatorname{supp}(d(\cdot, \cdot, t)) \subset K(a)+(0,-t) .
\end{aligned}
$$

A quick look at the Broadwell equations (4.1) shows that the collision term $r l$ has a damping effect on both $r$ and $l$; similarly $u d$ damps $u$ and $d$. The global existence condition (4.14) does not follow from global existence results known in the literature, even though it neglects these effects.

Proof of Theorem 2.1. We will show that the condition (4.5) implies (4.6). Let $N>0$ be such that

$$
0 \leq u_{1}(x, y, t), u_{2}(x, y, t), u_{3}(x, y, t), u_{4}(x, y, t) \leq N
$$

for all $x, y$ and for $0 \leq t \leq T$. Let

$$
N_{1}(t) \stackrel{\text { def }}{=} \sup _{x-y=t-a-\varepsilon} u_{1}(x, y, t) \quad \text { and } \quad N_{4}(t) \stackrel{\text { def }}{=} \sup _{x-y=t-a-\varepsilon} u_{4}(x, y, t)
$$


where $\varepsilon>0$. Observe that $N_{1}(t)$ and $N_{4}(t)$ are nonnegative and $N_{1}(0)=N_{4}(0)=0$. Since

$$
\begin{aligned}
u_{1}(x, y, t) & \leq u_{1}(x-t, y, 0)+\int_{0}^{t} u_{3}(x-t+s, y, s) u_{4}(x-t+s, y, s) d s \\
& \leq u_{1}(x-t, y, 0)+N \int_{0}^{t} u_{4}(x-t+s, y, s) d s, \\
u_{4}(x, y, t) & \leq u_{4}(x, y+t, 0)+\int_{0}^{t} u_{1}(x, y+t-s, s) u_{2}(x, y+t-s, s) d s \\
& \leq u_{4}(x, y+t, 0)+N \int_{0}^{t} u_{1}(x, y+t-s, s) d s
\end{aligned}
$$

we obtain (after taking the supremum over the line $x-y=t-a-\varepsilon$ ) that

$$
N_{1}(t) \leq N_{1}(0)+N \int_{0}^{t} N_{4}(s) d s
$$

and that

$$
N_{4}(t) \leq N_{4}(0)+N \int_{0}^{t} N_{1}(s) d s .
$$

Inequalities (4.20) and (4.21) together with the fact that $N_{1}(0)=N_{4}(0)=0$ imply that $N_{1}(t) \equiv N_{4}(t) \equiv 0$. Thus, $\operatorname{supp}[(r, l, u, d)(\cdot, \cdot, t)] \subset\{(x, y): x-y-t \leq a\}$. To show that $\operatorname{supp}[(r, l, u, d)(\cdot, \cdot, t)] \subset\{(x, y):-a \leq x-y-t\}$ we notice that the domain of dependence for points $(x, y, t)$ such that $-a \leq x-y-t$ lies entirely in the set, where $u_{1} \equiv u_{4} \equiv 0$.

We note that the same results are valid for numerical solutions of (1.3) and (3.1). One obtains that if $a M(0)<2$, and if the initial numerical data $(2.4)$ is bounded by $M(0)$ and supported on either $\Omega_{1}(a)$ or $\Omega_{2}(a)$, then the numerical solutions are bounded by the function

$$
M(t)=\min \left(\frac{M(0)}{1-t M(0)}, \frac{2 M(0)}{2-a M(0)}\right) .
$$

We also note that the above results can be easily extended to a large class of discreie velocity models of the Boltzmann equation. One may consider the following class of systems:

$$
\begin{aligned}
& \frac{\partial u_{i}}{\partial t}+V_{i} \cdot \nabla u_{i}=Q^{i}(\mathbf{u}), \quad i=1, \ldots, p \\
& \mathbf{u}(\mathbf{x}, 0)=\mathbf{u}_{0}(\mathbf{x})
\end{aligned}
$$

where $V_{i}, i=1,2, \ldots, p$, are constant vectors in $\mathbb{R}^{n}, \mathbf{u}=\left(u_{1}, u_{2}, \ldots, u_{p}\right), \mathbf{x}=\left(x_{1}, x_{2}, \ldots\right.$, $x_{n}$ ), and the collision terms $Q^{i}$ satisfy

$$
Q^{i}(\mathbf{u}) \leq \sum_{j, k} A_{j k}^{i} u_{j} u_{k}, \quad i=1, \ldots, p,
$$

where $A_{j k}^{i}$ are positive constants. 
We need to assume that there exists a unit vector $V$ and a number $1 \leq s \leq p$ such that

$$
V \cdot V_{i}<0 \text { for } i=1,2, \ldots, s-1 \text { and } V \cdot V_{i}>0 \text { for } i=s, s+1, \ldots, p
$$

and that

$$
A_{j k}^{i}=0 \text { if either } j<s \text { and } k<s \text { or } j \geq s \text { and } k \geq s .
$$

Let

$$
c_{1} \stackrel{\text { def }}{=} \min _{j \geq s} V \cdot V_{j} \quad \text { and } \quad c_{2} \stackrel{\text { def }}{=} \min _{j \leq s-1}\left|V \cdot V_{j}\right| .
$$

We show the following generalization of Theorem 4.1.

THEOREM 4.2. (a) Let $\mathbf{u}=\left(u_{1}, u_{2}, \ldots, u_{p}\right)$ be a uniformly bounded solution of (4.22) defined on $\mathbb{R}^{2} \times[0, T], 0 \leq u_{i}, i=1,2, \ldots, p$, and let the support of the initial data $\mathbf{u}_{0}$ be included in $\Omega_{V}(-a)=\{\mathbf{x}: \mathbf{x} \cdot V \geq-a\}, a>0$.

Then at times $0 \leq t \leq T$

$$
\operatorname{supp}\left[\left(u_{s}, u_{s+1}, \ldots, u_{p}\right)(\cdot, \cdot, t)\right] \subset \Omega_{V}\left(c_{1} t-a\right)=\Omega_{V}(-a)+t c_{1} V
$$

(b) If, in addition,

$$
\operatorname{supp}\left(\mathbf{u}_{0}\right) \subset \Omega_{-V}(-a)=\{\mathbf{x}: \mathbf{x} \cdot V \leq a\}
$$

then

$$
\operatorname{supp}\left[\left(u_{1}, u_{2}, \ldots, u_{s-1}\right)(\cdot, \cdot, t)\right] \subset \Omega_{-V}\left(a-c_{2} t\right)=\Omega_{-V}(-a)-t c_{2} V
$$

Thus, for $t \geq a /\left(c_{1}+c_{2}\right)$ the supports in (4.25) and (4.26) are disjoint and $\mathbf{u}$ satisfies

$$
\frac{\partial u_{i}}{\partial t}+V_{i} \cdot \nabla u_{i} \leq 0
$$

Proof. We will show (a). Without loss of generality we may assume that

$$
A_{j k}^{i}= \begin{cases}1 & \text { if } j<s \text { and } k \geq s \\ 0 & \text { otherwise. }\end{cases}
$$

Let $N$ be such that

$$
0 \leq u_{1}, u_{2}, \ldots, u_{p} \leq N
$$

Define

$$
v_{i} \stackrel{\text { def }}{=} V \cdot V_{i}, \quad i=1,2, \ldots, p .
$$

Similarly to the proof of Theorem 4.1 we let

$$
N_{i}(t, \varepsilon) \stackrel{\text { def }}{=} \sup _{V \cdot \mathbf{x}=-(a+\varepsilon)} u_{i}(\mathbf{x}+V t, t), \quad i=s, s+1, \ldots, p,
$$


and note that $N_{i}(0, \varepsilon)=0$, for all $\varepsilon>0$, and that $N_{i}(t, \varepsilon) \leq N$ for all $\varepsilon$ and $0 \leq t \leq T$. Condition (4.24) and (4.28) imply that for $i \geq s$

$$
u_{i}(\mathbf{x}, t) \leq u_{i}\left(\mathbf{x}-V_{i} t, 0\right)+N \sum_{j \geq s} \int_{0}^{t} u_{j}\left(\mathbf{x}-V_{i}\left(t-q_{1}\right), q_{1}\right) d q_{1}
$$

and, thus, that

$$
N_{i}(t, \varepsilon) \leq N \sum_{j \geq s} \int_{0}^{t} N_{j}\left(q_{1}, \varepsilon+\left(v_{i}-c_{1}\right)\left(t-q_{1}\right)\right) d q_{1} .
$$

We note that $\varepsilon+\left(v_{i}-c_{1}\right)(t-s)>0$ for all $\varepsilon>0$ and, therefore, that $N_{j}\left(0, \varepsilon+\left(v_{i}-c_{1}\right) t\right)=$ 0 . We plug the formulas $(4.31)_{j}$ into $(4.31)_{i}$ and obtain that

$$
N_{i}(t, \varepsilon) \leq N^{2} \sum_{j \geq s} \sum_{k \geq s} \int_{0}^{t} \int_{0}^{q_{1}} N_{k}\left(q_{2}, \varepsilon+\left(v_{i}-c_{1}\right)\left(t-q_{1}\right)+\left(v_{j}-c_{1}\right)\left(q_{1}-q_{2}\right)\right) d q_{2} d q_{1}
$$

and observe that $\varepsilon+\left(v_{i}-c_{1}\right)\left(t-q_{1}\right)+\left(v_{j}-c_{1}\right)\left(q_{1}-q_{2}\right)>0$ for $\varepsilon>0$ and $0 \leq q_{2} \leq q_{1} \leq t$.

Again we substitute $(4.31)_{k}$ into $(4.32)_{i}$ and obtain

$$
\begin{gathered}
N_{i}(t, \varepsilon) \leq N^{3} \sum_{j \geq s} \sum_{k \geq s} \sum_{l \geq s} \int_{0}^{t} \int_{0}^{q_{1}} \int_{0}^{q_{2}} N_{l}\left(q_{3}, \varepsilon+\left(v_{i}-c_{1}\right)\left(t-q_{1}\right)\right. \\
\left.+\left(v_{j}-c_{1}\right)\left(q_{1}-q_{2}\right)+\left(v_{k}-c_{1}\right)\left(q_{2}-q_{3}\right)\right) d q_{3} d q_{2} d q_{1}
\end{gathered}
$$

and so on.

Repeating the above procedure inductively we obtain, in the $n$th step, that $N_{i}(t, \varepsilon)$ is bounded by $(p-s+1)^{n}$ terms each less than $\frac{1}{n !} N^{n+1} t^{n}$. Thus

$$
N_{i}(t, \varepsilon) \leq N \frac{1}{n !}(p N t)^{n}, \quad n=1,2, \ldots
$$

Letting $n \rightarrow \infty$ yields that $N_{i}(t, \varepsilon)=0$ for $0 \leq t \leq T$ and $\varepsilon>0$ which completes the proof of our result.

Theorem 4.2 yields results on global existence, stability, and long-time behavior which generalize the results presented for the 2-D Broadwell model. These generalizations are straightforward and therefore we do not present them here.

\section{REFERENCES}

[1] T. Płatkowski and R. Illner, Discrete velocity models of the Boltzmann equation: A survey of mathematical aspects of the theory, SIAM Review 30, 213-255 (1988)

[2] R. Illner, Global existence results for discrete velocity models of the Boltzmann equation in several dimensions, J. Méch. Théor. Appl. 1, 611-622 (1982)

[3] R. Illner, Examples of non-bounded solutions in discrete kinetic theory, J. Méch. Théor. Appl. 5, 561-571 (1986)

[4] S. Kawashima, Global solution of the initial value problem for a discrete velocity model of the Boltzmann equation, Proc. Japan Acad. Ser. A. Math. Sci. 57, 1-24 (1981)

[5] R. Peszek, Convergence of numerical solutions to systems of semilinear hyperbolic equations, manuscript (1993)

[6] B. B. Mandelbrot, The Fractal Geometry of Nature, W. H. Freeman and Company, New York, 1983 